\title{
A strange case involving partial fracture of percutaneous transluminal coronary angioplasty wire and its successful retrieval
}

\author{
Santosh Kumar Sinha DM, Ramesh Thakur DM, Mukesh Jitendra Jha MD, Vinay Krishna MCh, \\ Chandra Mohan Varma DM, Pradyot Tiwari MD, Vikas Mishra MD, Ashutosh Kumar MD, Rupesh Sinha DM
}

SK Sinha, R Thakur, MH Jha, et al. A strange case involving partial fracture of percutaneous transluminal coronary angioplasty wire and its successful retrieval. Curr Res Cardiol 2015;2(4):199-201.

A 43-year-old woman underwent coronary angioplasty for refractory angina of a critical lesion of the proximal left anterior descending artery. After negotiating with spring-type wire (balance middle-weight wire), a loop was formed. Due to excessive manipulation to unloop the wire, it partially fractured at the shaft and coil junction. As the proximal section was retrieved, a thin elongated filament of spring coil also came out from the Y-connector while the remainder of the coil was still trapped in the lesion. Two balance middle-weight wires were then placed distally, and the proximal ends of both wires were inserted together in a torque device, which were firmly screwed and rotated 45 to 55 times in a circular pattern. During this rotational motion, the broken segment along with thin filament was tangled within these rescue wires and all three wires were removed together using tangling technique. Following this, angioplasty was performed successfully. After reviewing the literature using PubMed, to the authors' knowledge, this is the first incident of its kind to be reported.

Key Words: Partial fracture; Rescue wire; Shoulder point; Spring coil; Springtype wire

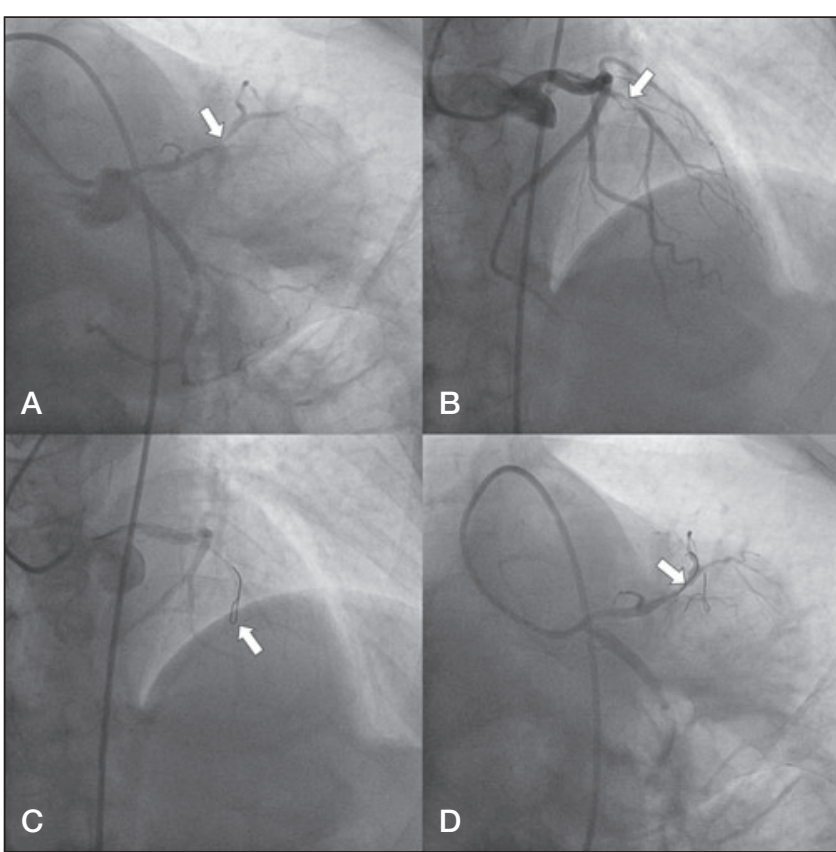

Figure 1) Critical lesion of the proximal left anterior descending artery (A and B). C A loop in tip of wire. D Fractured wire

using a 0.014 inch $190 \mathrm{~cm} \mathrm{Hi-Torque} \mathrm{BMW} \mathrm{wire} \mathrm{(Abbott} \mathrm{Vascular,}$ Santa Clara, USA) with some difficulty due to calcium. A loop was formed distally, which could not be further advanced (Figure 1C). Due to excessive manipulation when attempting to unloop it, a fracture occurred at the shoulder region (Figure 1D). As the proximal end was pulled, a thin filament of the spring also came out of the Y-connector confirming partial fracture of the wire (Figure 2). Two BMW wires were placed distally and the proximal ends of both wires Coronary Guide Catheter (Medtronic, USA). The lesion was crossed

Department of Cardiology, LPS Institute of Cardiology, GSVM Medical College, Kanpur, Uttar Pradesh, India

Correspondence: Dr Sinha Santosh Kumar, Faculty Department of Cardiology, LPS Institute of Cardiology, GSVM Medical College, Kanpur, Uttar

Pradesh, India 208002. Telephone 91-9670220088, fax 91-0512-2556199,2556521, e-mail fionasan@rediffmail.com 


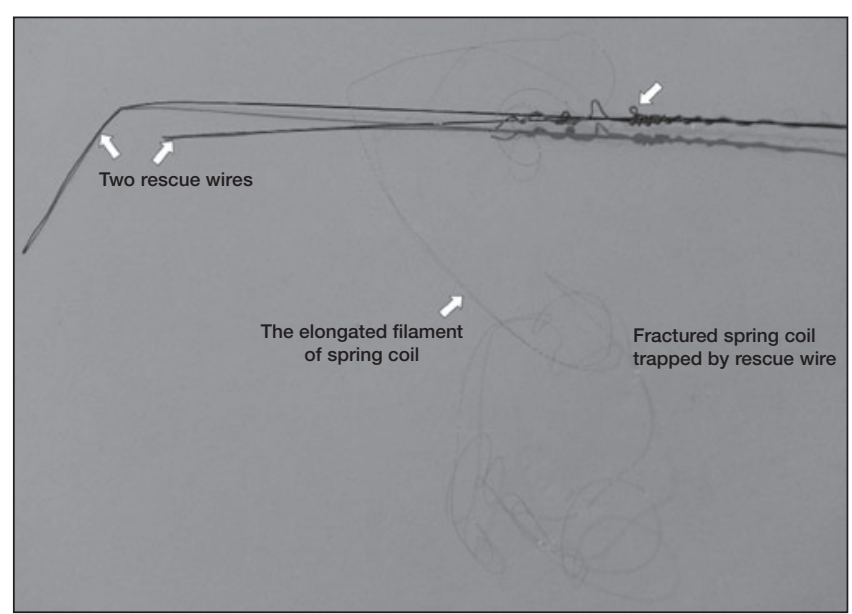

Figure 2) Partial fracture of the wire as thin filament is connected distally with coil

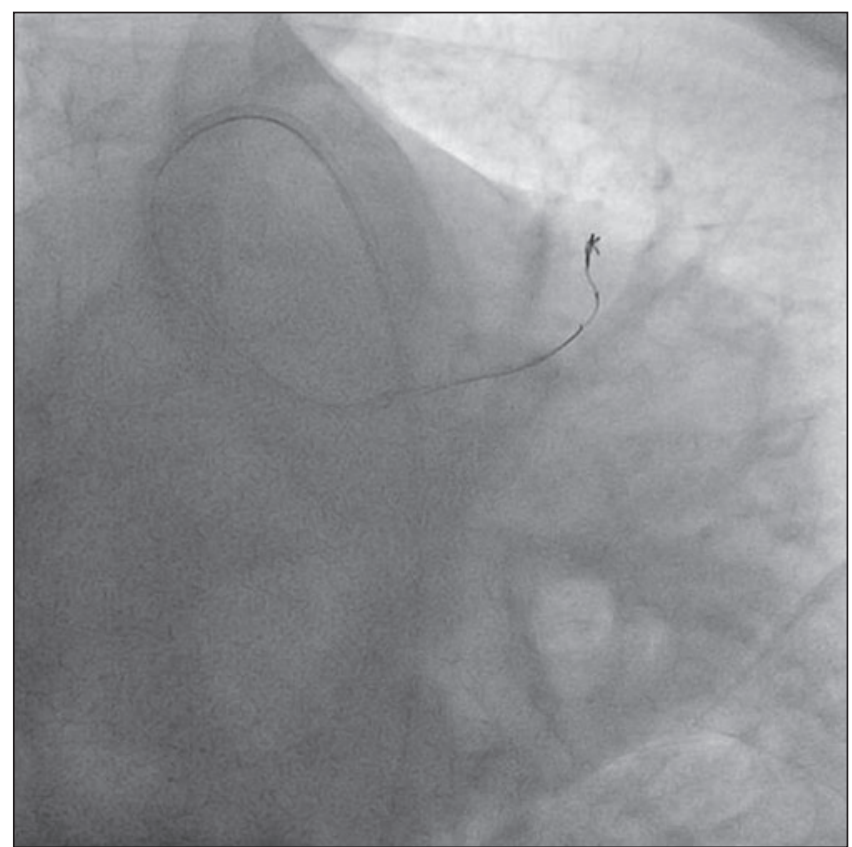

Figure 3) Twin rescue wire trapping fractured wire

were inserted together in a torque device, which were firmly screwed and rotated 45 to 55 times in a circular pattern to catch hold of the wire (Figure 3). During this rotational motion, the broken segment, along with thin filament, were tangled within these rescue wires and all three wires were removed together. Following this, the lesion was recrossed with a 0.014 inch $190 \mathrm{~cm} \mathrm{Hi-Torque} \mathrm{BMW} \mathrm{wire.} \mathrm{The}$ lesion was predilated with a $2 \times 10$ and $2.5 \times 10$ Sprinter Legend $\mathrm{Rx}$ balloon (Medtronic, USA). Xience Prime $3.0 \mathrm{~mm} \times 28 \mathrm{~mm}$ (Abbott Vascular, USA) was deployed in the proximal LAD at 12 atm with postprocedural thrombolysis in myocardial infarction (TIMI) III flow (Figure 4). The patient was discharged in stable condition with appropriate advice.

\section{DISCUSSION}

It is generally believed that the retained guidewire in the coronary tree may result in complications, such as emboli, thrombosis, dissection and rupture, and should be removed from the coronary system $(2,3)$. Most fractures are complete; the tip completely separates from the body of the wire. Various solutions have been described to solve the problem of a broken guidewire in a coronary artery, ranging from surgery to leaving the segment of wire in place in cases where it cannot be

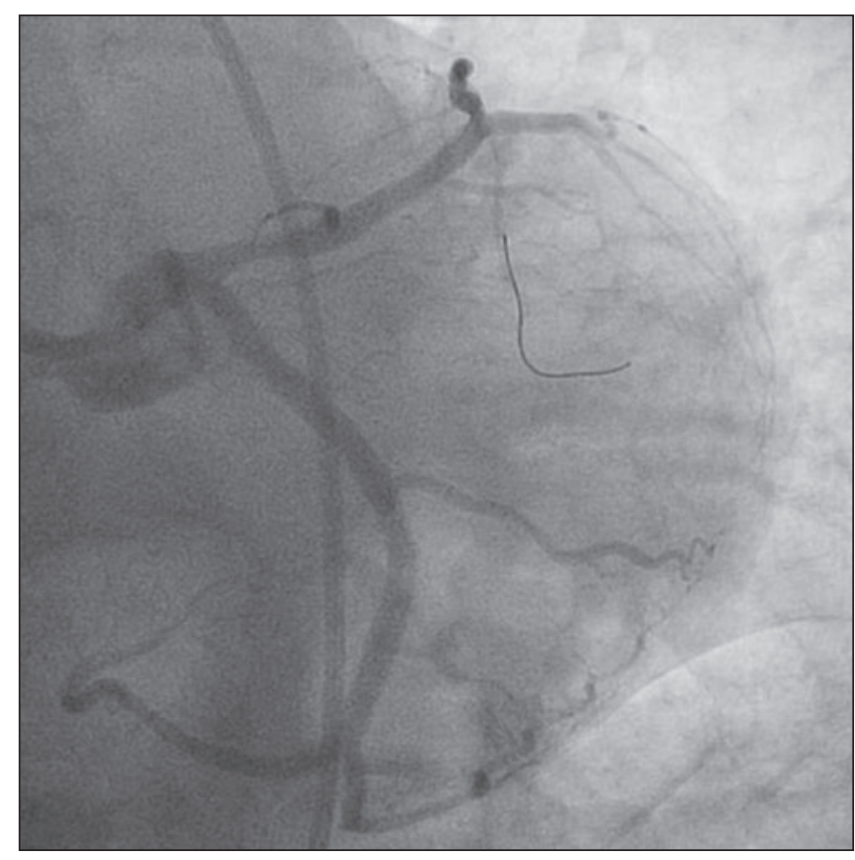

Figure 4) Poststenting left anterior descending artery

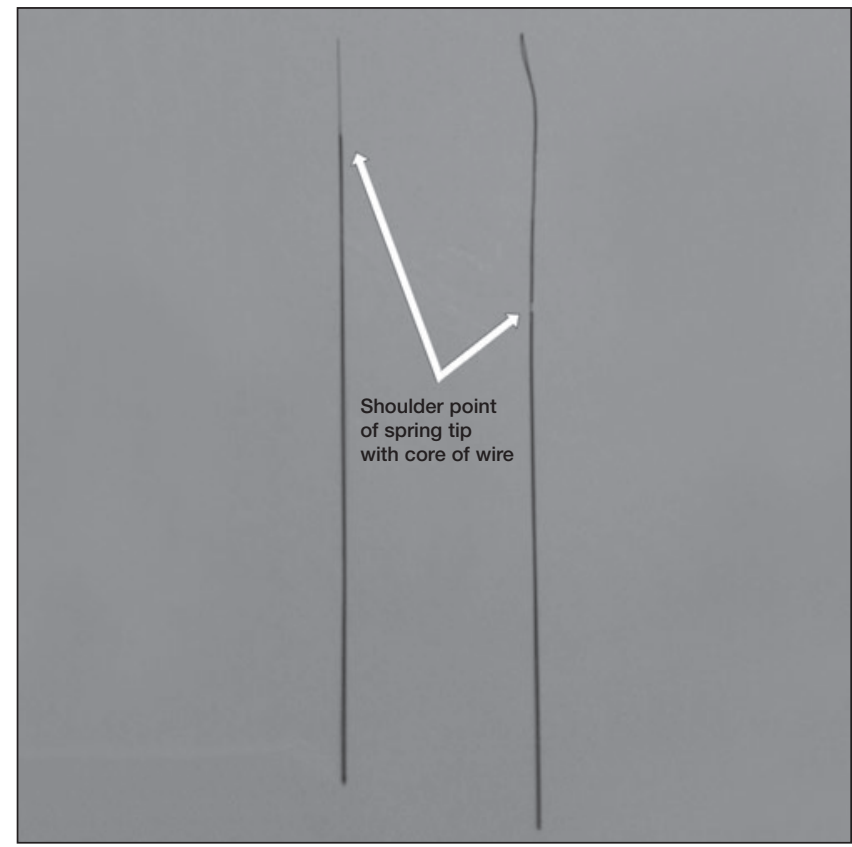

Figure 5) Design of balance middle-weight wire showing junction of body with spring coil

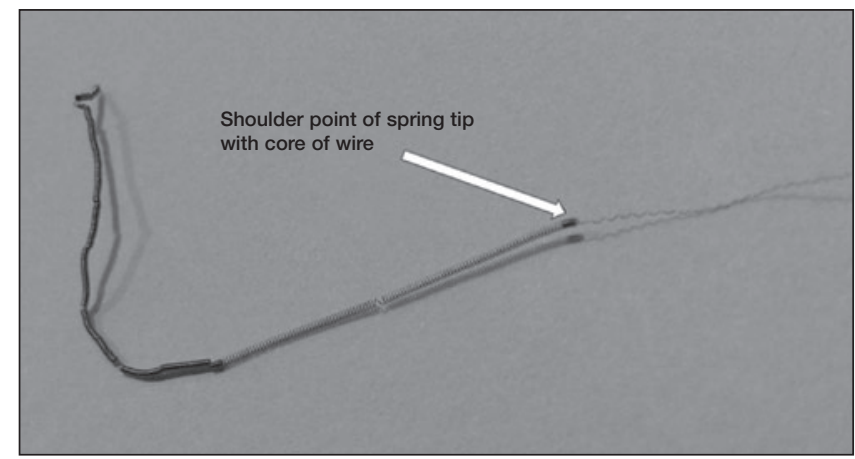

Figure 6) Thin filament extending from spring coil 
removed, such as in vessels that are already occluded or in very small branches; however, all efforts should be made to remove the guidewire percutaneously $(4,5)$. In our case, a partial fracture occurred because thin filament was still attached to the distal spring coil while the proximal end was removed from the Y-connector (Figures 5 and 6). This thin filament was lying across the left main to the mid LAD artery. Therefore, we decided to retrieve it. A simple technique was used because snare loop wire was not available. Two wires were used for the retrieval. Usually wire breaks at the shoulder point of the body with spring coil; however, our case was unique because it was a partial fracture. In BMW wire, the core does not extend up to the tip, so excessive turning may cause a fracture or, less commonly, a manufacturing defect may play a role. Vigorous twisting of the wire should always be avoided. In our case, the partial fracture was caused by trying to unloop the wire. To our knowledge, this is the first report of a partial fracture.

DISCLOSURES: The authors have no financial relationships or conflicts of interest to declare.

\section{REFERENCES}

1. Chang TM, Pellegrini D, Ostrovsky A, Marrangoni AG. Surgical management of entrapped percutaneous transluminal coronary angioplasty hardware. Tex Heart Inst J 2002;29:329-32.

2. Hartzler GO, Rutherford BD, McConahay DR. Retained percutaneous transluminal coronary angioplasty equipment components and their management. Am J Cardiol 1987;60:1260-4.

3. Sethi GK, Ferguson TB Jr, Miller G, Scott SM. Entrapment of broken guidewire in the left main coronary artery during percutaneous transluminal coronary angioplasty. Ann Thorac Surg 1989;47:455-7.

4. Patel T, Shah S, Pandya R, Sanghvi K, Fonseca K. Broken guidewire fragment: A simplified retrieval technique. Catheter Cardiovasc Interv 2000;51:483-6.

5. Demircan S, Yazici M, Durna K, Yasar E. Intracoronary guidewire emboli: A unique complication and retrieval of the wire. Cardiovasc Revasc Med 2008;9:278-80. 